Original Article

\title{
Platinum-Based Adjuvant Chemotherapy for Stage II and Stage III Squamous Cell Carcinoma of the Lung
}

\author{
Tetsuya Isaka, MD, ${ }^{1,2}$ Haruhiko Nakayama, MD, PhD, ${ }^{1}$ Tomoyuki Yokose, MD, PhD, ${ }^{3}$ \\ Hiroyuki Ito, MD, PhD, ${ }^{1}$ Kayoko Katayama, $\mathrm{PhD},{ }^{4}$ Kouzo Yamada, MD, PhD, ${ }^{5}$ \\ and Munetaka Masuda, $\mathrm{MD}, \mathrm{PhD}^{2}$
}

\begin{abstract}
Introduction: The efficacy of platinum-based adjuvant chemotherapy (PBAC) for pathological stage II and stage III squamous cell carcinoma (SCC) of the lung was analyzed retrospectively. Materials and Methods: The prognoses of 94 patients with stage II and stage III SCC with or without PBAC (more than three courses of cisplatin-, carboplatin-, and nedaplatinbased adjuvant chemotherapy) were compared.

Results: The mean observation period was 46.1 months. PBAC was not administered for the following reasons: $39(55.7 \%)$ patients had comorbidities, $25(35.7 \%)$ were older than 75 years, $19(27.1 \%)$ patients underwent surgery before the approval of PBAC, and 3 (4.3\%) patients could not continue PBAC ( $\leq 2$ cycles) because of adverse events. PBAC patients $(n=24)$ were significantly younger than non-PBAC patients $(n=70 ; 66.3$ vs 69.6 years old, respectively; $p=0.043$ ). Disease-free survival (DFS) did not differ between PBAC and non-PBAC patients $(55.0 \%$ and $67.1 \%$, respectively; $p=0.266)$. PBAC patients tended to have worse overall survival (OS) than non-PBAC patients $(56.1 \%$ and $70.2 \%$, respectively; $p=0.138)$. PBAC was not prognostic for OS (hazard ratio $(\mathrm{HR}), 2.11 ; 95 \%$ confidence interval $(\mathrm{CI}), \mathbf{0 . 8 2} \%-\mathbf{5 . 4 0 \%} ; \mathrm{p}=\mathbf{0 . 1 2 0})$.

Conclusion: PBAC did not improve the prognoses of patients with pathological stage II or stage III SCC in the single institution experience.
\end{abstract}

Keywords: platinum-based adjuvant chemotherapy, squamous cell carcinoma of the lung, disease-free survival, overall survival

\footnotetext{
${ }^{1}$ Department of Thoracic Surgery, Kanagawa Cancer Center, Yokohama, Kanagawa, Japan

${ }^{2}$ Department of Surgery, Yokohama City University, Yokohama, Kanagawa, Japan

${ }^{3}$ Department of Pathology, Kanagawa Cancer Center, Yokohama, Kanagawa, Japan

${ }^{4}$ Kanagawa Cancer Center, Research Institute Cancer Prevention \& Control Division, Kanagawa Cancer Center, Yokohama, Kanagawa, Japan

${ }^{5}$ Department of Thoracic Oncology, Kanagawa Cancer Center, Yokohama, Kanagawa, Japan
}

Received: June 29, 2016; Accepted: September 13, 2016 Corresponding author: Tetsuya Isaka MD. Department of Thoracic Surgery, Yokohama City University, Kanagawa Cancer Center, 2-3-2 Nakao, Asahi, Yokohama, Kanagawa 241-8515, Japan Email: 1401092k@yahoo.co.jp

(C)2017 The Editorial Committee of Annals of Thoracic and Cardiovascular Surgery. All rights reserved.

\section{Introduction}

Squamous cell carcinoma (SCC) of the lung accounts for $20 \%-30 \%$ of primary lung cancers and is the second most common histopathological type after pulmonary adenocarcinoma in Japan. ${ }^{1,2)}$ The prognoses of SCC and pulmonary adenocarcinoma differ according to lung cancer progression after complete resection. In stage I lung cancer, disease-free survival (DFS) and overall survival (OS) of patients with SCC are poorer than those of patients with pulmonary adenocarcinoma because adenocarcinoma in situ or minimally invasive adenocarcinoma confers good prognoses after curative resection. ${ }^{3)}$ Moreover, SCC is more frequent among elderly men who smoke, which might be related to non-cancer 
deaths. ${ }^{3,4)}$ However, the prognosis of resectable advanced SCC of stage II or higher after complete resection is better than that of pulmonary adenocarcinoma of the same stage. ${ }^{5,6)}$ Platinum-based doublet chemotherapy is commonly used for recurrent SCC, while various types of therapy, including molecular targeted drugs, are used for recurrent pulmonary adenocarcinoma. ${ }^{7-11)}$ Whether the favorable prognosis of patients with resectable advanced SCC is due to the less aggressive nature of the SCC tumor or the therapeutic effect of the chemotherapy on SCC remains unclear.

Based on several studies in unresectable advanced SCC, cisplatin + docetaxel is considered the standard first-line chemotherapy. ${ }^{11-13)}$ However, evidence for adjuvant chemotherapy to treat SCC remains unclear. Based on several clinical trials and the lung adjuvant cisplatin evaluation (LACE) pooled analysis, cisplatin-based chemotherapy improves OS for patients with non-smallcell lung cancer ${ }^{14-19)}$ although its efficacy for patients with SCC was not evident. Thus, in clinical practice, a cisplatin + vinorelbine regimen is used as adjuvant chemotherapy for SCC patients, ${ }^{20)}$ and carboplatin- or nedaplatin-based regimens are used for those who cannot tolerate cisplatin toxicity. This retrospective study aimed to examine the efficacy of platinum-based adjuvant chemotherapy (PBAC) for completely resected pathological stage II and stage III SCC.

\section{Materials and Methods}

\section{Patients}

From April 2002 to June 2015, 94 patients were treated with complete resection (pneumonectomy, lobectomy, or segmentectomy with negative surgical margin and lymph node dissection) for pathological stage II or stage III SCC. Patients who received neoadjuvant therapy and those who died within 3 months of surgery were excluded (one patient died of acute exacerbation of interstitial pneumonia). A pneumonectomy was performed depending on the location and expanse of the tumor. A segmentectomy was performed for high-risk patients who were considered intolerable to lobectomy. All other patients underwent lobectomy. Staging was based on the 7th Edition of the TNM Classification for Lung and Pleural Tumors.

\section{Definition of postoperative adjuvant chemotherapy and regimen}

Patients who received platinum doublet chemotherapy including cisplatin $\left(60-80 \mathrm{mg} / \mathrm{m}^{2}\right)$, carboplatin (an area
Table 1 Platinum-based adjuvant chemotherapy regimens

\begin{tabular}{lc} 
Platinum-based adjuvant chemotherapy regimen & 24 \\
Cisplatin + vinorelbine & 7 \\
Cisplatin + docetaxel & 1 \\
Cisplatin + TS-1 (tegafur, gimeracil, and oteracil & 1 \\
potassium) & \\
Carboplatin + paclitaxel & 4 \\
Carboplatin + vinorelbine & 1 \\
Carboplatin + TS-1 (tegafur, gimeracil, and & 1 \\
oteracil potassium) & \\
Nedaplatin + irinotecan & 9 \\
Discontinuation of adjuvant chemotherapy due to & 3 \\
side effects & \\
Carboplatin + paclitaxel & 2 \\
Cisplatin + vinorelbine & 1 \\
\hline
\end{tabular}

under the curve dose of $5-6 \mathrm{mg} / \mathrm{ml}$ per minute), and nedaplatin $\left(100 \mathrm{mg} / \mathrm{m}^{2}\right)$ regimens every 4 weeks for at least three cycles were included in the PBAC group. Concomitant drugs used along with platinating agents were vinorelbine $\left(25 \mathrm{mg} / \mathrm{m}^{2}\right.$ on days 1 and 8$)$, paclitaxel (200 $\mathrm{mg} / \mathrm{m}^{2}$ on day 1$)$, docetaxel $\left(60 \mathrm{mg} / \mathrm{m}^{2}\right.$ on day 1$)$, irinotecan $\left(50-60 \mathrm{mg} / \mathrm{m}^{2}\right.$ on days 1 and 8$)$, and TS-1 (tegafur, gimeracil, and oteracil potassium; $40 \mathrm{mg} / \mathrm{m}^{2}$ twice per day). Patients who ceased treatment within two cycles of PBAC due to adverse events were included in the non-PBAC group. PBAC regimens used for the patients enrolled in the study are shown in Table 1. All patients who underwent surgery before 2006 were included in the non-PBAC group because PBAC was not approved for SCC at stage II and stage III at our hospital before 2006 .

\section{Postoperative follow-up and recurrence pattern}

Patients received medical examinations every 3-6 months for the first to third years and every 6-12 months for fourth and fifth years after surgery. Computed tomography or positron emission tomography - computed tomography was performed at least once a year. When patients presented with recurrent cancer, cranial bone magnetic resonance imaging and positron emission tomography - computed tomography or bone scintigraphy were also conducted to determine the recurrence pattern (locoregional or distant). Locoregional recurrence was defined as a recurrence in the ipsilateral chest including dissemination at the mediastinum, supraclavicular, or cervical lymph nodes. Distant metastasis was defined as all recurrences other than locoregional recurrence, such as recurrence in the contralateral lung, brain, liver, adrenals, or bone. 
Table 2 Comparison of clinicopathological features between PBAC and non-PBAC patients

\begin{tabular}{|c|c|c|c|}
\hline & $\operatorname{PBAC}(+)(\mathrm{n}=24)$ & $\operatorname{PBAC}(-)(\mathrm{n}=70)$ & $\mathrm{p}$ values ${ }^{\mathrm{a}}$ \\
\hline Mean age (range) & $66.3(50-74)$ & $69.6(51-82)$ & $0.043^{b}$ \\
\hline Male $(\%)$ & $22(91.7)$ & $62(88.6)$ & 0.967 \\
\hline Current ex-smoker (\%) & $24(100)$ & $69(98.6)$ & 0.573 \\
\hline Comorbidity incidence (\%) & $11(45.8)$ & $39(55.7)$ & 0.403 \\
\hline Cardiac diseases & 0 & $7(10.0)$ & 0.246 \\
\hline Other cancers & $5(20.8)$ & $13(18.6)$ & 0.954 \\
\hline Chronic hepatitis & 0 & $4(5.7)$ & 0.541 \\
\hline Respiratory dysfunctions & $7(29.2)$ & $26(37.1)$ & 0.480 \\
\hline Tumor size $(\mathrm{mm})$ & 49.2 & 39.1 & $0.008^{b}$ \\
\hline \multicolumn{4}{|l|}{ Surgical procedures $(\%)$} \\
\hline Lobectomy & $23(95.8)$ & $61(87.1)$ & \\
\hline Pneumonectomy & $1(4.2)$ & $6(8.6)$ & \\
\hline Segmentectomy & 0 & $3(4.3)$ & 0.352 \\
\hline \multicolumn{4}{|l|}{ Pathological stage (\%) } \\
\hline p Stage IIA & $12(50.0)$ & $38(54.3)$ & \\
\hline p Stage IIB & $5(20.8)$ & $15(21.4)$ & \\
\hline p Stage IIIA & $7(29.2)$ & $17(24.3)$ & 0.891 \\
\hline Lymphatic invasion (\%) & $6(25.0)$ & $27(38.6)$ & 0.229 \\
\hline Vascular invasion (\%) & $20(83.3)$ & $53(75.7)$ & 0.625 \\
\hline Pleural invasion & $9(37.5)$ & $23(32.9)$ & 0.679 \\
\hline \multicolumn{4}{|l|}{ Pathological N status (\%) } \\
\hline pNO & $1(4.2)$ & $8(11.4)$ & \\
\hline $\mathrm{pN} 1$ & $18(75.0)$ & $47(67.1)$ & \\
\hline $\mathrm{pN} 2$ & $5(20.8)$ & $15(21.4)$ & 0.564 \\
\hline Recurrent (\%) & $6(25.0)$ & $15(21.4)$ & 0.717 \\
\hline Distant metastasis & $1(16.7)$ & $4(26.7)$ & \\
\hline Locoregional & $6(100)$ & $11(73.3)$ & 0.921 \\
\hline
\end{tabular}

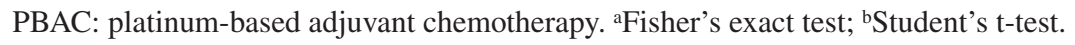

\section{Pathological examination}

Pathological diagnoses were made by pathological experts according to the 2004 World Health Organization classification. Tumor sections were routinely stained with hematoxylin and eosin as well as p40 or p63 to confirm SCC. Thyroid transcription factor 1 and napsin A or synaptophysin, CD56, and chromogranin A were stained to distinguish SCC from adenocarcinoma or neuroendocrine tumors, respectively, when necessary. Elastica-van Gieson or D2-40 staining was performed to evaluate vascular and pleural invasion or lymphatic invasion, respectively, when necessary.

\section{Statistical analysis}

Student's t-tests and Fisher's exact tests were used to analyze the continuous and categorical variables between PBAC and non-PBAC patients, respectively. Five-year DFS and OS were analyzed using the Kaplan-Meier method and compared between PBAC and non-PBAC patients using log-rank tests. The Cox proportional hazard regression model was used for multivariate analysis. A $p$ value $<0.05$ was considered statistically significant.
This study was approved by the Institutional Review Board at Kanagawa Cancer Center and was performed in accordance with the Declaration of Helsinki.

\section{Results}

The mean observation period for all patients was 46.1 months. Five-year DFS and OS of all patients were $64.2 \%$ and $67.2 \%$, respectively. Five-year OS of pathological stage IIA $(\mathrm{n}=50)$, IIB $(\mathrm{n}=20)$, and stage IIIA $(n=24)$ was $76.6 \%, 44.5 \%$, and $64.1 \%$, respectively.

In total, $24(25.5 \%)$ and $70(74.5 \%)$ patients were included in PBAC and non-PBAC groups, respectively. PBAC was not given for the following reasons: $39(55.7 \%)$ patients had comorbidities, 25 (35.7\%) patients were older than 75 years, $19(27.1 \%)$ patients were treated before 2006, and $3(4.3 \%)$ patients discontinued PBAC ( $\leq 2$ cycles) because of adverse events (some patients were not administered PBAC for multiple reasons). The regimens used for the three patients who discontinued PBAC within two cycles are also shown in Table 1. 
A. Disease-free survival

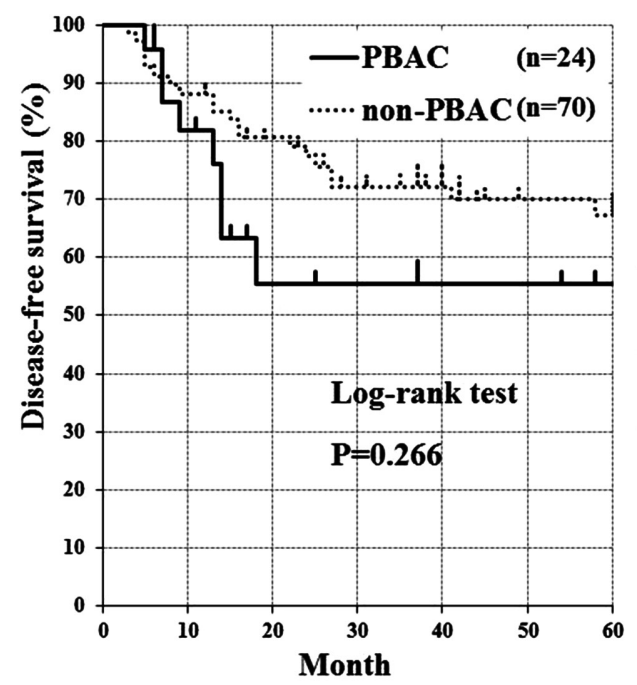

Patients at risk

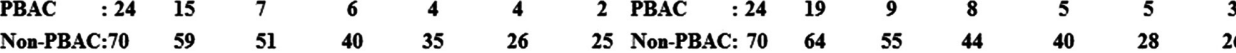

Fig. 1 DFS and OS for patients treated with or without PBAC. (A) DFS did not differ between PBAC and non-PBAC patients $(55.0 \%$ vs $67.1 \%$; $=0.266)$. (B) PBAC patients tended to have worse 5-year OS than non-PBAC patients (56.1\% vs $70.2 \%$; $=0.138)$. DFS: diseasefree survival; OS: overall survival; PBAC: platinum-based adjuvant chemotherapy
The clinicopathological backgrounds of PBAC and non-PBAC patients are shown in Table 2. PBAC patients were significantly younger than non-PBAC patients $(p=0.043)$. Tumors in PBAC patients were significantly larger than in non-PBAC patients $(\mathrm{p}=0.008)$. However, other clinicopathological backgrounds did not differ between PBAC and non-PBAC patients. The most common SCC recurrence pattern was local $(81.0 \%$ of patients with relapse), and the recurrence pattern did not differ between PBAC and non-PBAC patients.

DFS did not differ between PBAC and non-PBAC patients $(55.0 \%$ vs $67.1 \%$; $p=0.266$; Fig. 1A). PBAC patients tended to have worse OS as compared to nonPBAC patients $(56.1 \%$ vs $70.2 \%$; $p=0.138$; Fig. 1B). PBAC was not a prognostic factor for DFS by multivariate analysis (hazard ratio (HR), 1.42; $95 \%$ confidence interval (CI), 0.60-3.35; $\mathrm{p}=0.429$ ), and OS (HR, 2.11; 95\% CI, 0.82-5.40; $\mathrm{p}=0.120$; Table 3). OS did not differ between each platinum (cisplatin, carboplatin, or nedaplatin)-based regimen ( $p=0.284$; Fig. 2).

\section{Discussion}

This retrospective study did not demonstrate any positive effect of PBAC for patients after curative resection of stage II or stage III SCC. Because PBAC patients were younger and were considered more tolerant of chemotherapy, they were hypothesized to have better prognoses than non-PBAC patients. Indeed, there were no deaths in the PBAC group, whereas five deaths were observed in the non-PBAC group within 1 year of surgery (data not shown). However, as shown in Fig. 1B, OS curves intersected approximately 15 months after surgery, and the OS curve of the PBAC group fell below that of the non-PBAC group, suggesting a possible negative effect of PBAC on OS after a certain period of time. Moreover, the HR of the PBAC group was $2.11(\mathrm{p}=0.120)$ by multivariable analysis, which suggests that PBAC did not improve the prognoses of these patients, rather, it may have a negative impact on OS.

LACE meta-analysis has shown a significant OS benefit of postoperative cisplatin-based chemotherapy as compared to surgery alone (HR, 0.89 ; 95\% CI, 0.82-0.96; $\mathrm{p}=0.005$ ), and the absolute benefit of chemotherapy after 5 years was $5.4 \% .{ }^{19)}$ Moreover, the CALGB9633 study has demonstrated a survival benefit of postoperative carboplatin + paclitaxel for stage IB non-small-cell lung cancer for patients with tumor size of $4 \mathrm{~cm}$ or more (HR, 0.69; 95\% CI, 0.48-0.99; $\mathrm{p}=0.043){ }^{21)}$ The reason that PBAC was not beneficial in patients with stage II and stage III SCC remains unclear; however, the survival benefit of PBAC might be reduced by its detrimental effects on SCC patients. 
Table 3 Multivariate Cox proportional hazard regression model for DFS and OS

\begin{tabular}{|c|c|c|c|c|c|c|}
\hline \multirow{2}{*}{ Variable } & \multicolumn{3}{|c|}{ Multivariate analysis for DFS } & \multicolumn{3}{|c|}{ Multivariate analysis for OS } \\
\hline & HR & $95 \% \mathrm{CI}$ & $\mathrm{p}$ value & HR & $95 \% \mathrm{CI}$ & $\mathrm{p}$ value \\
\hline Age $(>65)$ & 0.72 & $0.34-1.50$ & 0.379 & 1.41 & $0.60-3.33$ & 0.437 \\
\hline Sex (male) & 1.82 & $0.41-7.98$ & 0.428 & 1.49 & $0.34-6.63$ & 0.600 \\
\hline Tumor size (>50 mm) & 1.36 & $0.53-3.49$ & 0.524 & 0.70 & $0.26-1.91$ & 0.487 \\
\hline Pathological stage (II and III) & 1.32 & $0.62-2.78$ & 0.473 & 1.91 & $0.95-3.88$ & 0.070 \\
\hline Differentiation (well, moderate, and poor) & 1.00 & $0.58-1.72$ & 0.990 & 0.86 & $0.46-1.61$ & 0.635 \\
\hline Lymphatic invasion $(+)$ & 0.96 & $0.46-2.03$ & 0.915 & 0.85 & $0.37-1.92$ & 0.695 \\
\hline Pleural invasion (+) & 1.30 & $0.58-2.88$ & 0.526 & 1.68 & $0.68-4.17$ & 0.264 \\
\hline PBAC (+) & 1.42 & $0.60-3.35$ & 0.429 & 2.11 & $0.82-5.40$ & 0.120 \\
\hline
\end{tabular}

DFS: disease-free survival; OS: overall survival; HR: hazard ratio; CI; confidence interval; PBAC: platinum-based adjuvant chemotherapy

A higher HRs of adjuvant chemotherapy were observed in SCC than in adenocarcinoma patients in a subgroup analysis of the International Adjuvant Lung Cancer Trial and in a LACE pooled analysis although they were not statistically significant. ${ }^{14,19)}$ The majority of studies have analyzed the efficacy of postoperative adjuvant chemotherapy for non-small-cell lung cancer in heterogeneous populations. Because patients with resectable advanced SCC inherently have more favorable prognoses than those with resectable advanced pulmonary adenocarcinoma, ${ }^{5,6)}$ adjuvant chemotherapy for SCC patients might be omissible.

There are various toxicities experienced in response to platinating agents. Renal and cardiovascular adverse events that are often life-threatening occur after cisplatin administration. ${ }^{22-25)}$ Although carboplatin-based chemotherapy is considered less toxic than cisplatin-based chemotherapy, thrombocytopenia, neutropenia, and nephrotoxicity often become problematic for patients during the course of chemotherapy. ${ }^{22,26)}$ Nedaplatin is a cisplatin derivative designed to reduce cisplatin toxicity and has potential antitumor effects against SCC in various organs. ${ }^{10,27)}$ Its use in combination with irinotecan confers a prognostic benefit for patients with SCC. ${ }^{11)}$ However, hematological adverse events are problematic in nedaplatin-based chemotherapy. ${ }^{10)}$ Because SCC frequently occurs in smoking elderly men who might have complicating respiratory disorders, vascular disorders, or other cancers, ${ }^{28)}$ these toxicities might negatively influence patient prognosis.

Long-term survival (beyond 5 years) benefits of adjuvant chemotherapy have not been shown;21,29) however, patients who did not smoke after adjuvant chemotherapy and had fewer comorbidities received a survival benefit from adjuvant chemotherapy. ${ }^{30)}$ Because 11 patients

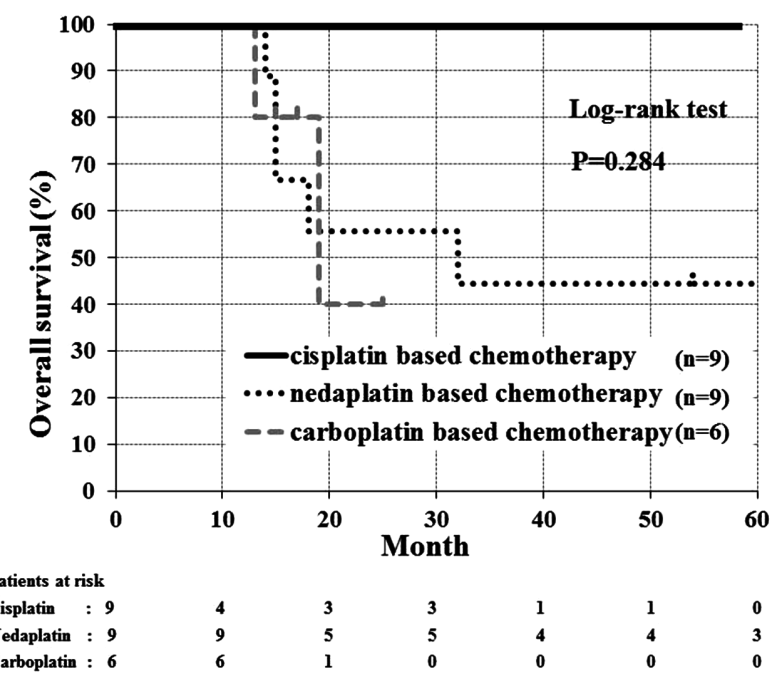

Fig. 2 Survival curves of patients receiving cisplatin-, carboplatin-, and nedaplatin-based regimens. All patients who received cisplatin-based chemotherapy remained alive for the duration of the observation period although no significant differences were detected in OS between patients who received carboplatin- and nedaplatin-based chemotherapy $(p=0.284)$. OS: overall survival

(45.8\%) had comorbidities in this study, these patients might not have received a survival benefit from PBAC. Moreover, it is uncertain whether patients in this study discontinued cigarette use.

There were some limitations in this study because it was a retrospective analysis with a small number of patients performed at a single institution. Patient backgrounds were not strictly matched between PBAC and non-PBAC groups, and various platinum-based chemotherapy regimens were included in this study. All of the patients who received cisplatin-based chemotherapy remained alive for the observation period; however, this study did not come to a definitive conclusion on 
whether cisplatin-based chemotherapy was superior to carboplatin- or nedaplatin-based chemotherapy because the number of the patients was too small (Fig. 2). A prospective study that includes a large patient population using a single platinum-based regimen is necessary to evaluate the efficacy and toxicity of PBAC for stage II and stage III SCC.

\section{Conclusion}

PBAC did not improve the prognoses of patients with pathological stage II or stage III SCC after curative resection of the lung, rather, it might have a negative impact on OS. A prospective study was required to evaluate whether PBAC was omissible for the patients with pathological stage II or stage III SCC.

\section{Disclosure Statement}

Tetsuya Isaka and other co-authors have no conflict of interest.

\section{References}

1) Sawabata N, Asamura H, Goya T, et al. Japanese Lung Cancer Registry Study: first prospective enrollment of a large number of surgical and nonsurgical cases in 2002. J Thorac Oncol 2010; 5: 1369-75.

2) Meza R, Meernik C, Jeon J, et al. Lung cancer incidence trends by gender, race and histology in the United States, 1973-2010. PLoS One 2015; 10: e0121323.

3) Maeda R, Yoshida J, Ishii G, et al. Prognostic impact of histology on early-stage non-small cell lung cancer. Chest 2011; 140: 135-45.

4) Fukui T, Taniguchi T, Kawaguchi K, et al. Comparisons of the clinicopathological features and survival outcomes between lung cancer patients with adenocarcinoma and squamous cell carcinoma. Gen Thorac Cardiovasc Surg 2015; 63: 507-13.

5) Khan OA, Fitzgerald JJ, Field ML, et al. Histological determinants of survival in completely resected T1-2N1M0 nonsmall cell cancer of the lung. Ann Thorac Surg 2004; 77: 1173-8.

6) Kawase A, Yoshida J, Ishii G, et al. Differences between squamous cell carcinoma and adenocarcinoma of the lung: are adenocarcinoma and squamous cell carcinoma prognostically equal? Jpn J Clin Oncol 2012; 42: 189-95. (in Japanese)

7) Sandler A, Gray R, Perry MC, et al. Paclitaxelcarboplatin alone or with bevacizumab for non-smallcell lung cancer. N Engl J Med 2006; 355: 2542-50.

8) Paz-Ares LG, de Marinis F, Dediu M, et al. PARAMOUNT: Final overall survival results of the phase III study of maintenance pemetrexed versus placebo immediately after induction treatment with pemetrexed plus cisplatin for advanced nonsquamous non-smallcell lung cancer. J Clin Oncol 2013; 31: 2895-902.

9) Mok TS, Wu YL, Thongprasert S, et al. Gefitinib or carboplatin-paclitaxel in pulmonary adenocarcinoma. N Engl J Med 2009; 361: 947-57.

10) Shukuya T, Yamanaka T, Seto T, et al. Nedaplatin plus docetaxel versus cisplatin plus docetaxel for advanced or relapsed squamous cell carcinoma of the lung (WJOG5208L): a randomised, open-label, phase 3 trial. Lancet Oncol 2015; 16: 1630-8.

11) Oshita F, Honda T, Murakami S, et al. Comparison of nedaplatin and irinotecan for patients with squamous and nonsquamous cell carcinoma of the lung: meta-analysis of four trials. J Thorac Oncol 2011; 6: 128-31.

12) Kubota K, Watanabe K, Kunitoh H, et al. Phase III randomized trial of docetaxel plus cisplatin versus vindesine plus cisplatin in patients with stage IV nonsmall-cell lung cancer: the Japanese Taxotere Lung Cancer Study Group. J Clin Oncol 2004; 22: 254-61.

13) Fossella F, Pereira JR, von Pawel J, et al. Randomized, multinational, phase III study of docetaxel plus platinum combinations versus vinorelbine plus cisplatin for advanced non-small-cell lung cancer: the TAX 326 study group. J Clin Oncol 2003; 21: 3016-24.

14) Arriagada R, Bergman B, Dunant A, et al. Cisplatinbased adjuvant chemotherapy in patients with completely resected non-small-cell lung cancer. N Engl J Med 2004; 350: 351-60.

15) Winton T, Livingston R, Johnson D, et al. Vinorelbine plus cisplatin vs. observation in resected non-smallcell lung cancer. N Engl J Med 2005; 352: 2589-97.

16) Douillard JY, Rosell R, De Lena M, et al. Adjuvant vinorelbine plus cisplatin versus observation in patients with completely resected stage IB-IIIA nonsmall-cell lung cancer (Adjuvant Navelbine International Trialist Association [ANITA]): a randomised controlled trial. Lancet Oncol 2006; 7: 719-27.

17) Scagliotti GV, Fossati R, Torri V, et al. Randomized study of adjuvant chemotherapy for completely resected stage I, II, or IIIA non-small-cell Lung cancer. J Natl Cancer Inst 2003; 95: 1453-61.

18) Waller D, Peake MD, Stephens RJ, et al. Chemotherapy for patients with non-small cell lung cancer: the surgical setting of the Big Lung Trial. Eur J Cardiothorac Surg 2004; 26: 173-82.

19) Pignon JP, Tribodet H, Scagliotti GV, et al. Lung adjuvant cisplatin evaluation: a pooled analysis by the LACE Collaborative Group. J Clin Oncol 2008; 26: 3552-9.

20) Douillard JY, Tribodet H, Aubert D, et al. Adjuvant cisplatin and vinorelbine for completely resected nonsmall cell lung cancer: subgroup analysis of the Lung Adjuvant Cisplatin Evaluation. J Thorac Oncol 2010; 5: 220-8. 
21) Strauss GM, Herndon JE 2nd, Maddaus MA, et al. Adjuvant paclitaxel plus carboplatin compared with observation in stage IB non-small-cell lung cancer: CALGB 9633 with the Cancer and Leukemia Group B, Radiation Therapy Oncology Group, and North Central Cancer Treatment Group Study Groups. J Clin Oncol 2008; 26: 5043-51.

22) Hotta K, Matsuo K, Ueoka H, et al. Meta-analysis of randomized clinical trials comparing Cisplatin to Carboplatin in patients with advanced non-small-cell lung cancer. J Clin Oncol 2004; 22: 3852-9.

23) Chaudhary UB, Haldas JR. Long-term complications of chemotherapy for germ cell tumours. Drugs 2003; 63: 1565-77.

24) Osanto S, Bukman A, Van Hoek F, et al. Long-term effects of chemotherapy in patients with testicular cancer. J Clin Oncol 1992; 10: 574-9.

25) Fosså SD, Gilbert E, Dores GM, et al. Noncancer causes of death in survivors of testicular cancer. J Natl Cancer Inst 2007; 99: 533-44.
26) Soria JC, Le Chevalier T. Is cisplatin still the best platinum compound in non-small-cell lung cancer? Ann Oncol 2002; 13: 1515-7.

27) Shimada M, Itamochi H, Kigawa J. Nedaplatin: a cisplatin derivative in cancer chemotherapy. Cancer Manag Res 2013; 5: 67-76.

28) Hirsch FR, Spreafico A, Novello S, et al. The prognostic and predictive role of histology in advanced non-small cell lung cancer: a literature review. J Thorac Oncol 2008; 3: 1468-81.

29) Arriagada R, Dunant A, Pignon JP, et al. Long-term results of the international adjuvant lung cancer trial evaluating adjuvant Cisplatin-based chemotherapy in resected lung cancer. J Clin Oncol 2010; 28: 35-42.

30) Butts CA, Ding K, Seymour L, et al. Randomized phase III trial of vinorelbine plus cisplatin compared with observation in completely resected stage IB and II non-small-cell lung cancer: updated survival analysis of JBR-10. J Clin Oncol 2010; 28: 29-34. 